\title{
SPECTRES MICROONDES, STRUCTURE ET CONSTANTES DE FORCE DE LA MOLÉCULE ClF
}

\author{
P. GOULET, R. JUREK et J. CHANUSSOT \\ Laboratoire de Spectroscopie Hertzienne (*), Faculté des Sciences MIPC \\ 6, bd Gabriel, 21000 Dijon, France
}

(Reçu le 9 décembre 1975, révisé le 20 janvier 1976, accepté le 23 janvier 1976)

\begin{abstract}
Résumé. - Les spectres rotationnels de $\mathrm{ClF}_{5}$ sont étudiés jusqu’à $210 \mathrm{GHz}$. Une nouvelle interprétation des transitions conduit à une meilleure approche des paramètres moléculaires. La géométrie de la molécule est déduite et les constantes de force calculées.
\end{abstract}

\begin{abstract}
Microwave spectra of $\mathrm{ClF}_{5}$ are studied up to $210 \mathrm{GHz}$. A new interpretation of the absorption lines gives a better determination of the rotational parameters. The molecular structure and force constants are given.
\end{abstract}

1. Introduction. - Sur la base des résultats de la première étude microonde de $\mathrm{ClF}_{5}$ [1], il n'est pas possible d'aboutir à une concordance acceptable entre la valeur calculée et la valeur mesurée du paramètre $D_{J K}$; de plus, la comparaison des paramètres de distorsion centrifuge relatifs à $\mathrm{ClF}_{5}$ et $\mathrm{BrF}_{5}[2,3]$ montre que si le paramètre $D_{J}$ diminue bien quand la masse de la molécule augmente, il n'en est pas de même pour $D_{J K}$. Ceci nous a conduits à remettre en cause la valeur de $D_{J K}$ et par là celle de $e q Q$. De nouvelles mesures ont donc été effectuées jusqu'à $210 \mathrm{GHz}$, l'identification reprise et les paramètres améliorés, ceci en vue de déterminer la géométrie et les constantes de force de $\mathrm{ClF}_{5}$.

2. Etude des spectres et paramètres obtenus. Les spectres sont obtenus à l'aide d'un spectromètre conventionnel de type vidéo travaillant à $-80^{\circ} \mathrm{C}$; les détails expérimentaux (en particulier le traitement des raies faibles par un moyenneur à échantillonnage) sont décrits par ailleurs [1]. D'une façon générale la largeur des raies observées est comprise entre 60 et $120 \mathrm{kHz}$ (la largeur Doppler à $70 \mathrm{GHz}$ est de $60 \mathrm{kHz}$; la précision d'un pointé de $10 \mathrm{kHz}$ ). Pour les deux molécules ${ }^{35} \mathrm{ClF}_{5}$ et ${ }^{37} \mathrm{ClF}_{5}$ les nouvelles transitions mesurées correspondent à $J=28 \rightarrow 29, J=29 \rightarrow 30$. Les paramètres moléculaires sont obtenus en utilisant une méthode de moindres carrés portant sur l'ensemble des 5 familles de transitions :

$J=9 \rightarrow 10, \quad 18 \rightarrow 19, \quad 19 \rightarrow 20$.

$$
28 \rightarrow 29, \quad 29 \rightarrow 30 \text {. }
$$

(*) Equipe de Recherche associée au C.N.R.S.
Soit au total un nombre de 150 points expérimentaux utilisés (respectivement 90 pour ${ }^{35} \mathrm{Cl}$ et 60 pour ${ }^{37} \mathrm{Cl}$ ). Nous donnons dans les tableaux I à $\mathrm{V}$ les résultats obtenus pour ${ }^{35} \mathrm{ClF}_{5}$ ainsi que la comparaison entre les fréquences mesurées et les fréquences calculées.

Les constantes rotationnelles sont données dans le tableau VI où la précision correspond à un intervalle de confiance à $99 \%$. On remarque que par rapport aux résultats donnés en [1] les paramètres moléculaires $D_{J K}$ et $e q Q$ ont varié; en particulier la valeur de $D_{J K}$ a doublé; la nouvelle valeur correspond bien cette fois aux prévisions théoriques. Bien que la précision affichée pour les paramètres soit moins bonne, les nouvelles mesures conduites en haute fréquence ont permis de corriger les erreurs d'identification de l'étude précédente [1] (transition $9 \rightarrow 10$ pour les raies $F=17 / 2$ et $19 / 2$; transition $18 \rightarrow 19$ et $19 \rightarrow 20$ pour les valeurs de $K \geqslant 7$ ). De là nous proposons un système plus satisfaisant pour les constantes rotationnelles nécessaires à la détermination de la géométrie et des constantes de force.

3. Structure moléculaire et constantes de force. L'étude des spectres d'absorption I.R. et Raman d'une molécule gazeuse de type $\mathrm{XY}_{5}$ (symétrie $\mathrm{C}_{4 \mathrm{v}}$ ) permet de déterminer la valeur numérique et la symétrie de ses 12 fréquences fondamentales de vibration [4]. Par ailleurs l'étude des spectres d'absorption hertziens pour l'état vibrationnel de base permet d'atteindre les constantes $B_{0}, D_{J} D_{J K}$ et $R_{6}$. Un développement théorique de l'hamiltonien jusqu'à l'ordre deux [5] explicite les paramètres rotationnels précédents en fonction des coefficients $a_{s \sigma}^{\alpha \beta}$ de variation des moments et produits d'inertie par rapport aux 
TABLEAU I

Transition $J=9 \rightarrow 10 d e{ }^{35} \mathrm{ClF}_{5}$

\begin{tabular}{|c|c|c|c|c|}
\hline \multirow{3}{*}{$\begin{array}{c}f_{\text {calc }} \\
(\mathrm{MHz})\end{array}$} & \multicolumn{4}{|c|}{ Transition $J=9 \rightarrow 10 \mathrm{de}^{35} \mathrm{ClF}_{5}$} \\
\hline & $\begin{array}{l}\text { Ident } \\
|K|\end{array}$ & $\begin{array}{l}\text { ation } \\
2 F\end{array}$ & $\begin{array}{c}f_{\text {mes }} \\
(\mathrm{MHz})\end{array}$ & $\begin{array}{l}f_{\mathrm{c}}-f_{\mathrm{m}} \\
(\mathrm{MHz})\end{array}$ \\
\hline & - & - & & - \\
\hline $\begin{array}{c}71000+ \\
0,129\end{array}$ & 9 & 15 & $\begin{array}{c}71000+ \\
0,222\end{array}$ & $-0,093$ \\
\hline 0,628 & 8 & 15 & 0,691 & $-0,063$ \\
\hline 1,069 & 7 & 15 & 1,137 & 0,004 \\
\hline 2,214 & 9 & 21 & & \\
\hline $\begin{array}{l}1,449 \\
1,451\end{array}$ & $\begin{array}{l}8 \\
6\end{array}$ & $\begin{array}{l}21 \\
15\end{array}$ & 1,503 & $-0,053$ \\
\hline 1,657 & 7 & 21 & 1,585 & $-0,072$ \\
\hline $\begin{array}{l}1,774 \\
1,836\end{array}$ & $\begin{array}{l}5 \\
6\end{array}$ & $\begin{array}{l}15 \\
21\end{array}$ & 1,815 & $-0,010$ \\
\hline 1,989 & 5 & 21 & & \\
\hline 2,038 & 4 & 15 & 2,083 & $-0,030$ \\
\hline
\end{tabular}

2,074

2,113

2,185

2,210

2,243

2,321

2,335

2,335

2,345

2,388

2,456

2,478

2,484

2,508

2,508

2,546

2,595

2,755

2,819

2,853

2,866

3,121

3,196

3,465

3,681

3,887

4,273

4,385

4,960

4,973

5,611

5,781

6,696

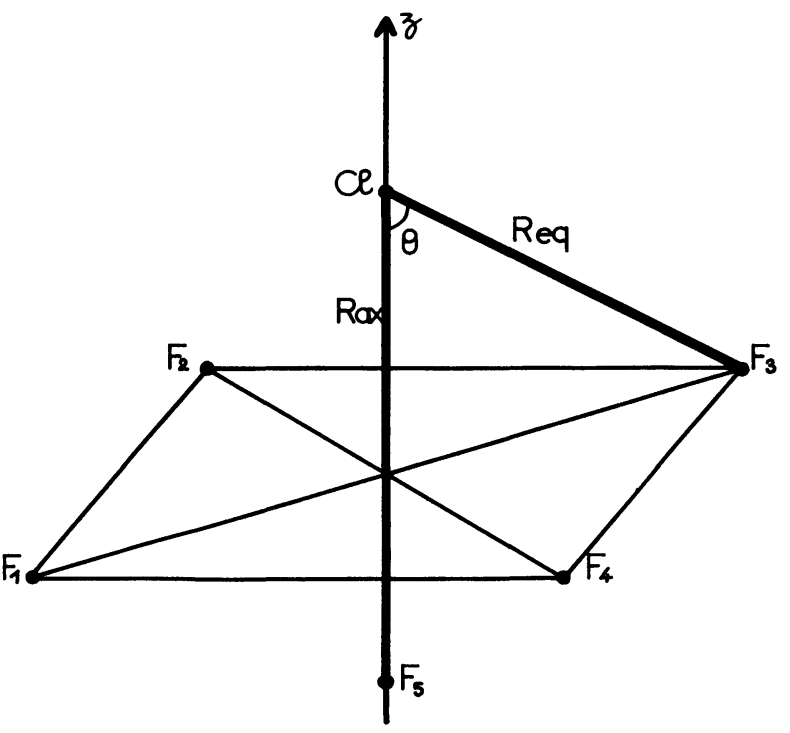

3.2 L'EXPRESSION DES COORDONNÉES NORMALES EN FONCTION DES DÉPLACEMENTS ATOMIQUES SYMÉTRISÉS. - Ce deuxième point devrait être réalisé par une diagonalisation de l'hamiltonien. Dans le formalisme de Wilson [6] on a l'hamiltonien sous la forme $\mathscr{H}=G F$, matrice $12 \times 12$. Dans la base des coordonnées symétriques les matrices $G$ et $F$ sont diagonales par blocs suivant la décomposition

$$
3 \mathrm{~A}_{1}+2 \mathrm{~B}_{1}+\mathrm{B}_{2}+3 \mathrm{E} \text {; }
$$

mais $\mathfrak{H}$ n'étant pas symétrique, il y a lieu de le symétriser en vue de sa diagonalisation : ceci sera réalisé à l'aide d'un changement de base tel que $G$ se réduise à l'identité $E$ soit $H^{\prime}=E F^{\prime}=F^{\prime}$. Soit $L$ une matrice de changement de base telle que

$$
H^{\prime}=L^{-1} \mathscr{H} L=L^{-1} G F L=L^{-1} G L^{+-1} L^{+} F L
$$

où $L^{+}$est la matrice transposée de $L$. On doit alors satisfaire à :

$$
\begin{aligned}
& E=L^{-1} G L^{+-1} \\
& F^{\prime}=L^{+} F L .
\end{aligned}
$$

On a ainsi symétrisé $\mathfrak{H}$ sans modifier ses valeurs propres $\lambda$ :

$$
\lambda E=\Lambda=M^{-1} H^{\prime} M
$$

soit en posant $L_{0}=L M, L_{0}^{-1} \dddot{H} L_{0}=\Lambda . L_{0}$ est donc la matrice de passage des coordonnées symétriques internes aux coordonnées normales et se déduit de $L$ par la rotation $M$. Le problème est donc de déterminer $L_{0}$ à partir de la connaissance de $L$; en première approximation nous prendrons $L_{0}=L$ ce qui revient à travailler dans un système de coordonnées pseudonormales en précisant toutefois que les calculs restent rigoureux pour la symétrie $B_{2}$ pour laquelle $\left(L_{0}\right)_{\mathbf{B}_{2}} \equiv(L)_{\mathbf{B}_{2}}$.

Pour cette raison, le calcul de $R_{6}$ [5] qui ne dépend coordonnées normales $Q_{s \sigma}$; ceux-ci s'expriment en fonction des coefficients $l_{\text {iso }}^{\alpha}$ qui relient les déplacements atomiques pondérés aux coordonnées normales [5]. Les calculs numériques correspondants nécessitent pour la molécule la connaissance de :

3.1 LA GÉOMÉTRIE (distances interatomiques et angles de valence). 
TABleaU II

Transition $J=18 \rightarrow 19 d e^{35} \mathrm{ClF}_{5}$

\begin{tabular}{|c|c|c|c|c|c|c|c|c|c|}
\hline \multirow{3}{*}{$\begin{array}{c}f_{\text {calc }} \\
(\mathrm{MHz})\end{array}$} & \multicolumn{2}{|c|}{ Identification } & \multirow{2}{*}{$\begin{array}{c}f_{\text {mes }} \\
(\mathrm{MHz})\end{array}$} & \multirow{2}{*}{$\begin{array}{c}f_{\mathrm{c}}-f_{\mathrm{m}} \\
(\mathrm{MHz})\end{array}$} & \multirow{2}{*}{$\begin{array}{c}f_{\text {calc }} \\
(\mathbf{M H z})\end{array}$} & \multicolumn{2}{|c|}{ Identification } & \multirow{2}{*}{$\begin{array}{c}f_{\text {mes }} \\
(\mathrm{MHz})\end{array}$} & \multirow{2}{*}{$\begin{array}{c}f_{\mathrm{c}}-f_{\mathrm{m}} \\
(\mathrm{MHz})\end{array}$} \\
\hline & $2 F$ & $|K|$ & & & & $2 F$ & $|K|$ & & \\
\hline & - & - & & - & - & - & - & & \\
\hline $\begin{array}{c}134800+ \\
88.058\end{array}$ & & & $134800+$ & & $\begin{array}{l}91,823 \\
91,918\end{array}$ & 33 & 11 & & \\
\hline 88,098 & 37 & & & & 91,829 & 35 & & 91,762 & 0,010 \\
\hline $\begin{array}{l}88,100 \\
88,140\end{array}$ & $\begin{array}{l}33 \\
35\end{array}$ & $2\}$ & 88,103 & $-0,004$ & 91,876 & 37 & 9 & & \\
\hline $\begin{array}{l}88,140 \\
89,392\end{array}$ & 39 & & & & 92,277 & 33 & 12 & & \\
\hline $\begin{array}{l}89,392 \\
89,02\end{array}$ & 37 & & & & 92,398 & 39 & 12 & 92303 & 0.078 \\
\hline 89,439 & 33 & 0 & & & $\begin{array}{l}92,389 \\
92459\end{array}$ & $\begin{array}{l}35 \\
37\end{array}$ & 10 & , 2,J0J & $0,0 / 0$ \\
\hline 89,439 & 35 & & & & $\begin{array}{l}92,439 \\
92,770\end{array}$ & 33 & & & \\
\hline $\begin{array}{l}89,413 \\
89,423\end{array}$ & 39 & & & & $\begin{array}{l}92,110 \\
92,920\end{array}$ & 39 & 13 & & \\
\hline $\begin{array}{l}89,423 \\
89,458\end{array}$ & 33 & 1 & & & 93,009 & $35 ?$ & & 92,841 & 0,110 \\
\hline 89,468 & 35 & & & & 93,103 & 37 & 11 & & \\
\hline 89,580 & 39 & & 89.561 & 0.056 & 93,302 & 33 & 14 & 93,419 & $-0,026$ \\
\hline 89,616 & 33 & 3 & $0 \%, 001$ & & 93,484 & 39 & 14 & & $-0,0<0$ \\
\hline $\begin{array}{l}89,668 \\
89,704\end{array}$ & $\begin{array}{l}37 \\
35\end{array}$ & & & & $\begin{array}{l}93,687 \\
93,809\end{array}$ & $\begin{array}{l}35 \\
37\end{array}$ & 12 & & \\
\hline 89,726 & $39)$ & & & & 94,873 & 33 & & & \\
\hline 89,754 & 33 & 4 & & & 94,089 & 39 & 15 & & \\
\hline $\begin{array}{l}89,883 \\
89.911\end{array}$ & $\begin{array}{l}37 \\
35\end{array}$ & & & & 94,425 & 35 & 13 & & \\
\hline $\begin{array}{l}89,911 \\
89,914\end{array}$ & 397 & & & & 94,575 & 37 & 13 & & \\
\hline $\begin{array}{l}89,914 \\
89,931\end{array}$ & 33 & 5 & & & $\begin{array}{l}94,484 \\
94,736\end{array}$ & $\begin{array}{l}33 \\
39\end{array}$ & 16 & & \\
\hline $\begin{array}{l}90,143 \\
90,148\end{array}$ & $\begin{array}{l}39 \\
33\end{array}$ & 6 & & & 95,222 & 35 & 14 & & \\
\hline 90,159 & 37 & 5 & 90,172 & $-0,015$ & $\begin{array}{l}95,403 \\
95,135\end{array}$ & $33\}$ & & & \\
\hline 90,176 & 35 & & & & 95,425 & 39 & 17 & & \\
\hline $\begin{array}{l}90,404 \\
90,415\end{array}$ & $\begin{array}{l}33 \\
39\end{array}$ & 7 & & & $\begin{array}{l}95,825 \\
96156\end{array}$ & $\left.\begin{array}{l}33 \\
39\end{array}\right\}$ & 18 & & \\
\hline 90,496 & 37 & & 90,340 & 0,116 & 96,077 & $35\}$ & & & \\
\hline 90,501 & 35 & 6 & & & 96,293 & 37 & 15 & & \\
\hline $\begin{array}{l}90,700 \\
90,728\end{array}$ & $\begin{array}{l}33 \\
39\end{array}$ & 8 & 90,704 & 0,010 & $\begin{array}{l}96,992 \\
97,244\end{array}$ & $\left.\begin{array}{l}35 \\
37\end{array}\right\}$ & 16 & & \\
\hline $\begin{array}{l}90,884 \\
90,895\end{array}$ & $\begin{array}{l}35 \\
37\end{array}$ & 7 & & & $\begin{array}{l}97,966 \\
98,256\end{array}$ & $\left.\begin{array}{l}35 \\
37\end{array}\right\}$ & 17 & & \\
\hline 90,892 & 39 & & & & 98,998 & 35\{ & & & \\
\hline 90,932 & 37 & $2+1$ & & & 99,330 & 37 & 18 & & \\
\hline 90,934 & 33 & 27 & & & & Eca & noyen & $22 \mathrm{MHz}$ & \\
\hline 90,974 & 35 & & 91.252 & -0110 & & & ype & $66 \mathrm{MHz}$ & \\
\hline $\begin{array}{l}91,035 \\
91,083\end{array}$ & $\begin{array}{l}33 \\
39\end{array}$ & 9 & & $-0,110$ & & & & & \\
\hline $\begin{array}{l}91,327 \\
91,355\end{array}$ & $\begin{array}{l}35 \\
37\end{array}$ & 8 & & & & & & & \\
\hline 91,410 & 33 & 10 & & & & & & & \\
\hline 91,479 & 39 & 10 & & & & & & & \\
\hline
\end{tabular}


TABleau III

Transition $J=19 \rightarrow 20 \mathrm{de}^{35} \mathrm{ClF}_{5}$

\begin{tabular}{|c|c|c|c|c|c|c|c|c|c|}
\hline \multirow{2}{*}{$\begin{array}{c}f_{\text {calc }} \\
(\mathrm{MHz})\end{array}$} & \multicolumn{2}{|c|}{ Identification } & \multirow{2}{*}{$\begin{array}{c}f_{\text {mes }} \\
(\mathrm{MHz})\end{array}$} & \multirow{2}{*}{$\begin{array}{c}f_{\mathrm{c}}-f_{\mathrm{m}} \\
(\mathrm{MHz})\end{array}$} & \multirow{2}{*}{$\begin{array}{c}f_{\text {calc }} \\
(\mathrm{MHz})\end{array}$} & \multicolumn{2}{|c|}{ Identification } & \multirow{2}{*}{$\begin{array}{c}f_{\text {mes }} \\
(\mathrm{MHz})\end{array}$} & \multirow{2}{*}{$\begin{array}{l}f_{\mathrm{c}}-f_{\mathrm{m}} \\
(\mathrm{MHz})\end{array}$} \\
\hline & $2 F$ & $|K|$ & & & & $2 F$ & $|K|$ & & \\
\hline $141 \overline{900}+$ & - & 一 & $\overline{\overline{0}}$ & 一 & - & - & - & - & - \\
\hline $\begin{array}{c}141900+ \\
84,891\end{array}$ & 41 & & $141900+$ & & $\begin{array}{l}88,945 \\
88,980\end{array}$ & $\left.\begin{array}{l}37 \\
39\end{array}\right\}$ & 9 & & \\
\hline 84,925 & 39 & $2^{-}$ & & & $\begin{array}{l}80,700 \\
89,140\end{array}$ & $35\}$ & & & \\
\hline $\begin{array}{l}84,929 \\
84,863\end{array}$ & $\begin{array}{l}35 \\
37\end{array}$ & 2 & 84,898 & 0,029 & 89,212 & $41\}$ & 11 & 89,180 & $-0,004$ \\
\hline 86,453 & 41 & & & & $\begin{array}{l}89,520 \\
89,573\end{array}$ & $\left.\begin{array}{l}37 \\
39\end{array}\right\}$ & 10 & & \\
\hline $\begin{array}{l}86,453 \\
86,495\end{array}$ & $\begin{array}{l}39 \\
35\end{array}$ & 0 & & & 89,642 & 35 & & 89,527 & 0,091 \\
\hline $\begin{array}{l}86,495 \\
86,495\end{array}$ & $\begin{array}{l}35 \\
37\end{array}$ & & & & 89,737 & 41 & 12 & & \\
\hline $\begin{array}{l}86,476 \\
86,484\end{array}$ & $\begin{array}{l}41 \\
39\end{array}$ & & & & $\begin{array}{l}90,156 \\
90,228\end{array}$ & $\begin{array}{l}37 \\
39\end{array}$ & 11 & & \\
\hline $\begin{array}{l}86,484 \\
86,517\end{array}$ & 35 & 1 & & & 90,189 & 35 & & 90,165 & 0,055 \\
\hline 86,525 & 37 & & & & 90,307 & 41 & 13 & & \\
\hline $\begin{array}{l}86,658 \\
86,692\end{array}$ & $\begin{array}{l}41 \\
35\end{array}$ & & 86,733 & $-0,044$ & $\begin{array}{l}90,779 \\
90,923\end{array}$ & $\left.\begin{array}{l}35 \\
41\end{array}\right\}$ & 14 & & \\
\hline 86,734 & 39 & 3 & & & 90,851 & 37 & 12 & 90,847 & 0,028 \\
\hline 86,767 & 37 & & & & 90,946 & 39 & 12 & & \\
\hline $\begin{array}{l}86,818 \\
86,845\end{array}$ & $\begin{array}{l}41 \\
35\end{array}$ & & & & 91,412 & 35 & 15 & & \\
\hline 86,952 & 39 & 4 & & & 91,584 & 41 & & 91,576 & 0,006 \\
\hline 86,979 & 37 & & & & $\begin{array}{l}91,608 \\
91,726\end{array}$ & $\left.\begin{array}{l}37 \\
39\end{array}\right\}$ & 13 & & \\
\hline $\begin{array}{l}87,023 \\
87,041\end{array}$ & $\begin{array}{l}41 \\
35\end{array}$ & 5 & & & $\begin{array}{l}92,090 \\
92,291\end{array}$ & $\left.\begin{array}{l}35 \\
41\end{array}\right\}$ & 16 & & \\
\hline $\begin{array}{l}87,233 \\
87,251\end{array}$ & $\begin{array}{l}39 \\
37\end{array}$ & 5 & & & 92,425 & 37 & 14 & 92,355 & $-0,011$ \\
\hline 87,274 & 41 & 6 & 87,287 & $-0,027$ & 92,568 & 39 & & & \\
\hline 87,282 & 35 & 0 & & & $\begin{array}{l}92,811 \\
93,041\end{array}$ & $\begin{array}{l}35 \\
41\end{array}$ & 17 & & \\
\hline $\begin{array}{l}87,566 \\
87,570\end{array}$ & $\begin{array}{l}35 \\
41\end{array}$ & 7 & & & 93,302 & 37 & 15 & 93,065 & 0,092 \\
\hline 87,576 & 39 & 6 & 87,442 & 0,132 & 93,473 & 39 & & & \\
\hline $\begin{array}{l}87,584 \\
87,894\end{array}$ & $\begin{array}{l}37 \\
35\end{array}$ & 0 & & & $\begin{array}{l}93,576 \\
93,841\end{array}$ & $\begin{array}{l}35 \\
41\end{array}$ & 18 & & \\
\hline $\begin{array}{l}87,894 \\
87,912\end{array}$ & $\begin{array}{l}35 \\
41\end{array}$ & 8 & 87829 & 0112 & $\begin{array}{l}94,240 \\
94,440\end{array}$ & $\begin{array}{l}35 \\
41\end{array}$ & 16 & 94302 & 0,053 \\
\hline $\begin{array}{l}87,977 \\
87,982\end{array}$ & $\left.\begin{array}{l}37 \\
39\end{array}\right\}$ & 7 & (1), & 0,112 & 94,385 & 35 & 19 & 94,302 & 0,053 \\
\hline 88,197 & 41 & & & & 94,685 & $41\}$ & 19 & & \\
\hline 88,231 & 39 & $2^{+}$ & & & 95,238 & 37 & 17 & 95,357 & $-0,003$ \\
\hline $\begin{array}{l}88,235 \\
88,269\end{array}$ & $\begin{array}{l}35 \\
37\end{array}$ & & & & 95,470 & $39\}$ & & & 0,000 \\
\hline 88,265 & 35 & & 88,343 & $-0,046$ & 96,297 & 37 & $18\}$ & 96,288 & 0,009 \\
\hline 88,300 & 41 & 9 & & & 96,562 & $39\}$ & $18\}$ & 96,585 & $-0,023$ \\
\hline 88,431 & 37 & & & & 97,416 & $37\}$ & $19\}$ & 97,379 & 0,037 \\
\hline 88,450 & 39 & 8 & & & $97,716^{\prime \prime}$ & $39\}$ & $19 ?$ & 97,812 & $-0,096$ \\
\hline $\begin{array}{l}88,681 \\
88,733\end{array}$ & $\left.\begin{array}{l}35 \\
41\end{array}\right\}$ & 10 & 88,691 & 0,016 & & $\begin{array}{l}\text { Ecar } \\
\text { Ecar }\end{array}$ & $\begin{array}{l}\text { noyen } \\
\text { jpe }\end{array}$ & $\begin{array}{l}20 \mathrm{MHz} \\
51 \mathrm{MHz}\end{array}$ & \\
\hline
\end{tabular}


Tableau IV

Transition $J=28 \rightarrow 29 \mathrm{de}^{35} \mathrm{ClF}_{5}$

\begin{tabular}{|c|c|c|c|c|c|c|c|c|c|}
\hline \multirow{2}{*}{$\begin{array}{c}f_{\text {calc }} \\
(\mathrm{MHz})\end{array}$} & \multicolumn{2}{|c|}{ Identification } & \multirow{2}{*}{$\begin{array}{c}f_{\text {mes }} \\
(\mathrm{MHz})\end{array}$} & \multirow{2}{*}{$\begin{array}{c}f_{\mathrm{c}}-f_{\mathrm{m}} \\
(\mathrm{MHz})\end{array}$} & \multirow{2}{*}{$\begin{array}{c}f_{\text {calc }} \\
(\mathrm{MHz})\end{array}$} & \multicolumn{2}{|c|}{ Identification } & \multirow{2}{*}{$\begin{array}{c}f_{\text {mes }} \\
(\mathrm{MHz})\end{array}$} & \multirow{2}{*}{$\begin{array}{c}f_{\mathrm{c}}-f_{\mathrm{m}} \\
(\mathrm{MHz})\end{array}$} \\
\hline & $2 F$ & $|K|$ & & & & $2 F$ & $|K|$ & & \\
\hline $205800+$ & & - & $205800+$ & & 49,642 & $53\}$ & & $40,0=$ & 0031 \\
\hline 36,452 & 59 & & & & 49,671 & $59\}$ & $15\}$ & 49,625 & 0,031 \\
\hline $\begin{array}{l}36,463 \\
36,482\end{array}$ & $\begin{array}{l}57 \\
55\end{array}$ & $2^{-}$ & 36,450 & 0,017 & $\begin{array}{l}50,261 \\
50,289\end{array}$ & $\left.\begin{array}{l}55 \\
57\end{array}\right\}$ & 15 & & \\
\hline 36,472 & 53 & & & & 50,790 & 53 & & & \\
\hline $\begin{array}{l}41,292 \\
41,292\end{array}$ & 59 & & & & 50,825 & 59 & 167 & & \\
\hline $\begin{array}{l}41,292 \\
41,312\end{array}$ & $\begin{array}{l}31 \\
55\end{array}$ & 0 & & & $\begin{array}{l}51,494 \\
51,529\end{array}$ & $\begin{array}{l}55 \\
57\end{array}$ & & 51,636 & $-0,185$ \\
\hline 41,312 & 53 & & & & 52,012 & $53\}$ & & & \\
\hline 41,329 & 59 & & & & 52,054 & $59\}$ & 17 & & \\
\hline $\begin{array}{l}41,332 \\
41,352\end{array}$ & $\begin{array}{l}57 \\
55\end{array}$ & 1 & & & $\begin{array}{l}52,807 \\
52,849\end{array}$ & $\left.\begin{array}{l}55 \\
57\end{array}\right\}$ & $17\}$ & 52,794 & 0,034 \\
\hline 41,349 & 53 & & & & $\begin{array}{l}5,, 049 \\
53,308\end{array}$ & $53\}$ & & & \\
\hline $\begin{array}{l}41,627 \\
41,652\end{array}$ & $\begin{array}{l}59 \\
57\end{array}$ & & & & 53,357 & $59\}$ & 18 & & \\
\hline $\begin{array}{l}41,632 \\
41,670\end{array}$ & $\begin{array}{l}51 \\
55\end{array}$ & 3 & 41,757 & $-0,063$ & 54,199 & 55 & $18\}$ & 54,278 & $-0,054$ \\
\hline 41,645 & 53 & & & & & $57 \mathrm{~J}$ & 10 & & , \\
\hline 41,888 & 59 & & & & $\begin{array}{r}54,678 \\
55775\end{array}$ & $\left.\begin{array}{c}53 \\
50\end{array}\right]$ & & & \\
\hline $\begin{array}{l}41,932 \\
41,948\end{array}$ & $\begin{array}{l}57 \\
55\end{array}$ & 4 & & & $\begin{array}{l}54,735 \\
55671\end{array}$ & $\left.\begin{array}{l}59 \\
55\end{array}\right\}$ & 19 & 55,302 & $-0,099$ \\
\hline 41,904 & 53 & & & & 55,728 & 57 & & & \\
\hline 42,223 & 59 & & & & 56,122 & 53 & 20 & & \\
\hline 42,292 & 57 & 5 & & & 56,187 & $59\}$ & 20 & & \\
\hline $\begin{array}{l}42,306 \\
42,237\end{array}$ & 53 & & & & $\begin{array}{l}57,222 \\
55720\end{array}$ & 55 & 20 & 57,262 & $-0,007$ \\
\hline 42,632 & 59 & & & & 57,640 & $53\}$ & & & \\
\hline 42,645 & 53 & 6 & & & 57,714 & $59\}$ & 21 & & \\
\hline $\begin{array}{l}42,731 \\
42,744\end{array}$ & $\begin{array}{l}51 \\
55\end{array}$ & & & & 58,853 & $55\}$ & 21 & & \\
\hline 43,116 & 59 & & 42,795 & 0,143 & 58,927 & $57\}$ & 21 & 58,767 & 0,123 \\
\hline 43,126 & 53 & 7 & & & 59,232 & 53\{ & 22 & & \\
\hline 43,251 & 57 & 7 & & & 59,315 & $59 \int$ & 22 & & \\
\hline 43,261 & 55 & & & & 60,563 & $55\}$ & $2 ?$ & & \\
\hline 43,675 & 59 & & & & 60,647 & $57\}$ & 22 & & \\
\hline 43,681 & 53 & 8 & & & 60,898 & 53\{ & 13 & 60,674 & 0,100 \\
\hline 43,851 & 57 & 0 & & & 60,991 & $59\}$ & 23 & & \\
\hline 43,857 & 55 & & 44,006 & 0,087 & 62,353 & $55\}$ & 23 & & \\
\hline $\begin{array}{l}44,308 \\
44,311\end{array}$ & $\left.\begin{array}{l}59 \\
53\end{array}\right]$ & & & & 62,446 & $57 \int$ & 20 & 62,219 & 0,325 \\
\hline $\begin{array}{l}44,311 \\
44,531\end{array}$ & 57 & 9 & & & $\begin{array}{l}62,638 \\
62,741\end{array}$ & 53 & 24 & & \\
\hline 44,534 & 55 & & & & & $59]$ & & & \\
\hline 45,014 & 53 & & & & 64,222 & $\left.\begin{array}{r}55 \\
57\end{array}\right\}$ & 24 & & \\
\hline $\begin{array}{l}45,016 \\
45,289\end{array}$ & $\begin{array}{l}59 \\
55\end{array}$ & 10 & & & $\begin{array}{l}64,326 \\
64,452\end{array}$ & $53\}$ & & 64,307 & 0,084 \\
\hline $\begin{array}{l}45,289 \\
45,291\end{array}$ & 57 & & 15637 & & $\begin{array}{l}04,422 \\
64,566\end{array}$ & $\left.\begin{array}{l}53 \\
59\end{array}\right\}$ & 25 & 1 & \\
\hline 45,792 & 53 & & 45,031 & $-0,080$ & 66,171 & $55\}$ & & & \\
\hline 45,797 & 59 & 11 & & & 66,285 & $57 \int$ & 25 & 66100 & 0117 \\
\hline $\begin{array}{l}46,125 \\
46,131\end{array}$ & $\begin{array}{l}55 \\
57\end{array}$ & & & & 66,341 & $59\}$ & 26 & 00,198 & $0, \Pi 11$ \\
\hline 46,430 & 59 & & & & $\begin{array}{l}66,465 \\
68,200\end{array}$ & $\begin{array}{l}53\} \\
55\}\end{array}$ & & & \\
\hline 46,441 & 57 & $2^{+}$ & & & $\begin{array}{l}68,325 \\
68,20\end{array}$ & $57\}$ & 26 & & \\
\hline 46,449 & 53 & & 46,558 & $-0,045$ & 68,303 & $53\}$ & & 68,290 & 0,027 \\
\hline 46,460 & $\begin{array}{l}55 \\
52\end{array}$ & & & & 68,439 & $59\}$ & 27 & & \\
\hline $\begin{array}{l}46,643 \\
46,654\end{array}$ & 59 & 12 & & & 70,308 & $55\}$ & 27 & & \\
\hline 47,039 & 55 & 12 & & & $\begin{array}{l}70,444 \\
70,339\end{array}$ & 57) & & 70,385 & 0,010 \\
\hline 47,050 & 57 & & 47,358 & & $\begin{array}{l}17,349 \\
70,487\end{array}$ & $\left.\begin{array}{l}53 \\
59\end{array}\right\}$ & 28 & & \\
\hline $\begin{array}{l}47,569 \\
47,585\end{array}$ & $\begin{array}{l}53 \\
59\end{array}$ & 13 & & & 72,496 & 55\{ & & & \\
\hline 48,034 & 55 & & & & 72,643 & $57\}$ & 28 & 72,645 & $-0,075$ \\
\hline 48,050 & 57 & 13 & & & & & ye & & \\
\hline $\begin{array}{l}48,569 \\
48,591\end{array}$ & $\begin{array}{l}53 \\
59\end{array}$ & 14 & 48,767 & 0,187 & & & type & $10 \mathrm{kHz}$ & \\
\hline 49,108 & 55 & 14 & & & & & & & \\
\hline 49,130 & 57 & 14 & & & & & & & \\
\hline
\end{tabular}


TABLEAU $\mathrm{V}$

Tranvition $J=29 \rightarrow 30 d{ }^{3 .}{ }^{35} \mathrm{ClF}_{5}$

\begin{tabular}{|c|c|c|c|c|c|c|c|c|c|}
\hline \multirow{2}{*}{$\begin{array}{c}f_{\text {calc }} \\
(\mathrm{MHz})\end{array}$} & \multicolumn{2}{|c|}{ Identification } & \multirow{2}{*}{$\begin{array}{c}f_{\text {mes }} \\
(\mathrm{MHz})\end{array}$} & \multirow{2}{*}{$\begin{array}{l}f_{\mathrm{c}}-f_{\mathrm{m}} \\
(\mathrm{MHz})\end{array}$} & \multirow{2}{*}{$\begin{array}{c}f_{\text {calc }} \\
(\mathrm{MHz})\end{array}$} & \multicolumn{2}{|c|}{ Identification } & \multirow{2}{*}{$\begin{array}{c}f_{\text {mes }} \\
(\mathrm{MHz})\end{array}$} & \multirow{2}{*}{$\begin{array}{c}f_{\mathrm{c}}-f_{\mathrm{m}} \\
(\mathrm{MHz})\end{array}$} \\
\hline & $2 F$ & $|K|$ & & & & $2 F$ & $|K|$ & & \\
\hline $212900+$ & - & - & $212 \overline{900}+$ & - & $4 \overline{1.911}$ & $\overline{57}$ ? & - & - & - \\
\hline 28,491 & 61 & \multirow{4}{*}{$2^{-}$} & \multirow{4}{*}{28,547} & \multirow{4}{*}{$-0,042$} & 41,929 & $59\}$ & 14 & & \multirow{4}{*}{0,151} \\
\hline 28,501 & 59 & & & & 42,540 & 55 & & \multirow[t]{3}{*}{42,377} & \\
\hline 28,509 & 55 & & & & 42,564 & 61 & 15 & & \\
\hline 28,519 & 57 & & & & 43,099 & 57 & 101 & & \\
\hline 33,859 & 61 & \multirow{3}{*}{0} & & & 43,123 & 59 & & \multirow{3}{*}{43,702} & \\
\hline $\begin{array}{l}33,859 \\
33,878\end{array}$ & $\begin{array}{l}59 \\
57\end{array}$ & & & & $\begin{array}{l}43,734 \\
43,763\end{array}$ & $\left.\begin{array}{l}55 \\
61\end{array}\right\}$ & 16 & & \multirow{2}{*}{0,047} \\
\hline 33,878 & 55 & & & & $\begin{array}{l}4,105 \\
44,370\end{array}$ & $57\}$ & & & \\
\hline 33,898 & 61 & \multirow{3}{*}{1} & & & 44,399 & 59 & 16 & 44,506 & $-0,122$ \\
\hline $\begin{array}{l}33,900 \\
33,916\end{array}$ & $\begin{array}{l}59 \\
55\end{array}$ & & & & 45,004 & 55 & & \multirow[b]{2}{*}{45,130} & \\
\hline $\begin{array}{l}33,916 \\
33,919\end{array}$ & $\begin{array}{l}55 \\
57\end{array}$ & & & & 45,040 & $61\}$ & 17 & & 0,070 \\
\hline 34,207 & 61 & & & & $\begin{array}{l}45,722 \\
45,758\end{array}$ & $\begin{array}{l}57 \\
59\end{array}$ & 17 & & \\
\hline $\begin{array}{l}34,224 \\
34,230\end{array}$ & $\begin{array}{l}55 \\
59\end{array}$ & 3 & 34,263 & 0,012 & 46,352 & 55\{ & & 45,941 & 0,116 \\
\hline $\begin{array}{l}34,230 \\
34,247\end{array}$ & 57 & & & & 46,394 & $61\}$ & 18 & & \\
\hline 34,478 & 61 & & & & 47,157 & 57 & 18 & & \\
\hline 34,494 & 55 & 4 & & & 47,199 & 59 & 10 & 47,389 & \\
\hline 34,518 & 59 & & & & 47,776 & 55 & & $4 /, 389$ & 0,100 \\
\hline 34,534 & 57 & & & & 47,825 & 61 & 19 & & \\
\hline $\begin{array}{l}34,826 \\
34,840\end{array}$ & $\left.\begin{array}{l}61 \\
55\end{array}\right]$ & & & & 48,673 & 57 & 19 & & \\
\hline 34,888 & 59 & 5 & & & 48,722 & $59\}$ & & & \\
\hline 34,902 & 57 & & & & $\begin{array}{l}49,278 \\
49,334\end{array}$ & $\left.\begin{array}{l}55 \\
61\end{array}\right\}$ & 20 & & \\
\hline $\begin{array}{l}35,252 \\
35,264\end{array}$ & $\left.\begin{array}{l}61 \\
55\end{array}\right]$ & & & & 50,272 & $57 ?$ & 20 & & \\
\hline 35,341 & 59 & 6 & & & 50,328 & 5)! & 20 & 50,379 & $-0,079$ \\
\hline $\begin{array}{l}35,353 \\
35,755\end{array}$ & 57 & & 35.552 & & 50,856 & 55 & & & \\
\hline $\begin{array}{l}35,755 \\
35,764\end{array}$ & $\left.\begin{array}{l}61 \\
55\end{array}\right)$ & & 35,332 & 0,009 & $\begin{array}{r}50,920 \\
5105\end{array}$ & $\begin{array}{l}61 \\
57\end{array}$ & 21 & & \\
\hline 35,877 & 59 & 7 & & & $\begin{array}{l}51,952 \\
52,016\end{array}$ & 59 & & 51,807 & 0,000 \\
\hline 35,886 & 57 & & & & 52,512 & $55\}$ & 22 & & \\
\hline $\begin{array}{l}36,335 \\
36,342\end{array}$ & $\begin{array}{l}61 \\
55\end{array}$ & & & & 52,584 & 61 & 22 & & \\
\hline 36,494 & $\begin{array}{l}55 \\
59\end{array}$ & 8 & & & 53,714 & 57 & 22 & & \\
\hline 36,501 & 57 & & & & $\begin{array}{l}53,786 \\
54,245\end{array}$ & $\begin{array}{l}59 \\
55\end{array}$ & & 54,041 & $-0,024$ \\
\hline 36,993 & 61 & & 36,647 & 0,109 & 54,325 & 61 & 23 & & \\
\hline $\begin{array}{l}36,996 \\
37104\end{array}$ & 55 & 9 & & & 55,559 & 57 & & & \\
\hline $\begin{array}{l}37,194 \\
37,198\end{array}$ & $\begin{array}{l}59 \\
57\end{array}$ & & & & 55,699 & 59 & 23 & & \\
\hline 37,728 & 61 & & & & $\begin{array}{l}56,054 \\
56,143\end{array}$ & $\begin{array}{l}55 \\
61\end{array}$ & 24 & & \\
\hline $\begin{array}{l}37,728 \\
37,996\end{array}$ & $\begin{array}{l}55 \\
59\end{array}$ & 10 & & & 57,485 & 57 & & & \\
\hline $\begin{array}{l}37,996 \\
37,996\end{array}$ & $\begin{array}{l}59 \\
57\end{array}$ & & & & 57,574 & 59 & 24 & & \\
\hline $\begin{array}{l}38,536 \\
38,540\end{array}$ & 55 & & 38,239 & 0,036 & 57,941 & 55 & 25 & 57,735 & 0,025 \\
\hline $\begin{array}{l}38,540 \\
38,837\end{array}$ & $\begin{array}{l}61 \\
57\end{array}$ & 11 & & & 58,039 & 61 & 20 & & \\
\hline $\begin{array}{l}38,831 \\
38,841\end{array}$ & $\begin{array}{l}57 \\
59\end{array}$ & & & & 59,493 & 59 & 25 & & \\
\hline 39,422 & 55 & & & & $\begin{array}{r}59,591 \\
59,904\end{array}$ & 57 & & 59,715 & 0,035 \\
\hline 39,430 & 61 & 12 & & & $\begin{array}{l}39,904 \\
60,012\end{array}$ & 61 & 26 & & \\
\hline $\begin{array}{l}39,780 \\
39,788\end{array}$ & $\begin{array}{l}57 \\
59\end{array}$ & & & & 61,584 & 57 & 26 & & \\
\hline $\begin{array}{l}39, / 80 \\
39,537\end{array}$ & 61 & & 39,740 & & 61,691 & 59 & 20 & 61,925 & $-0,105$ \\
\hline 39,547 & $\begin{array}{l}59 \\
55\end{array}$ & $2^{+}$ & $3 y, 140$ & 0,000 & $\begin{array}{l}61,945 \\
62,062\end{array}$ & $\begin{array}{l}55 \\
61\end{array}$ & 27 & & \\
\hline $\begin{array}{l}39,555 \\
39,565\end{array}$ & $\begin{array}{l}53 \\
57\end{array}$ & & & & 63,756 & $\begin{array}{l}57 \\
59\end{array}$ & 27 & & \\
\hline $\begin{array}{l}40,384 \\
40,397\end{array}$ & $\left.\begin{array}{l}55 \\
61\end{array}\right\}$ & 13 & & & $\begin{array}{l}63,873 \\
64,062\end{array}$ & 55 & & 63,912 & 0,051 \\
\hline & 57 & & & & 64,160 & 61 & 28 & & \\
\hline 40,817 & 59 & 13 & 41,197 & $-0,075$ & $\begin{array}{l}66,010 \\
66,138\end{array}$ & $\begin{array}{l}57 \\
59\end{array}$ & 28 & & \\
\hline 41,424 & $\left.\begin{array}{l}55 \\
61\end{array}\right\}$ & 14 & & & $\begin{array}{l}60,138 \\
66,257\end{array}$ & 55 & & 66,329 & $-0,128$ \\
\hline 41,442 & & & & & 66,395 & 61 & 29 & & \\
\hline & $\begin{array}{l}\text { Eca } \\
\text { Eca }\end{array}$ & $\begin{array}{l}\text { moyen } \\
\text { type }\end{array}$ & $\begin{array}{l}5 \mathrm{kHz} \\
7 \mathrm{kHz}\end{array}$ & & $\begin{array}{l}68,346 \\
68,484\end{array}$ & $\left.\begin{array}{l}57 \\
59\end{array}\right\}$ & 29 & 68,612 & $-0,197$ \\
\hline
\end{tabular}


TABLEAU VI Constantes rotationnelles

$B_{0} \quad(\mathrm{MHz})$

$D_{J} \quad(\mathrm{~Hz})$

$D_{J K}(\mathrm{~Hz})$

$e Q q(\mathrm{MHz})$

$R_{6},(\mathrm{~Hz})$
${ }^{35} \mathrm{ClF}_{5} \quad \pm$ à $99 \%$

$3550,273 \pm \overline{0,006}$

$764 \pm 6$

$-664 \quad+5$

$44,67 \pm 2,50$

$51,2 \pm 0,8$
${ }^{37} \mathrm{ClF}_{5} \quad \pm$ à $99 \%$

$3 \overline{545,888} \pm \overline{0}, 007$

$755 \pm 6$

$-586 \pm 16$

$35,40 \pm 3,00$

$51,2 \pm 4,0$ que des coordonnées de symétrie $B_{1}$ et $B_{2}$ [7] est quasi exact ; par contre les études de $D_{J}\left(\mathrm{~A}_{1}, \mathrm{~B}_{1}\right.$ et $\left.\mathrm{B}_{2}\right)$ et $D_{J K}\left(\mathrm{~A}_{1}, \mathrm{~B}_{1}, \mathrm{~B}_{2}\right.$ et $\left.\mathrm{E}\right)$ serviront à préciser a posteriori, à partir des éléments de $L$, les éléments de $L_{0}$ par application de la matrice $M$ [8].

4. Résultats. - La méthode de Costain [9] (approximation de structure $r_{\mathrm{s}}$ et $\theta_{\mathrm{s}}$ en lieu et place de $r_{0}$

TABLEAU VII

$\begin{array}{rrrr}4,892 & 0,240 & -2,174 & \\ 0,240 & 3,204 & -0,303 & \mathrm{~A}_{1} \\ -2,174 & -0,303 & 4,225 & \end{array}$

Matrice $G\left(\times 1,66 \cdot 10^{-4} \mathrm{~kg}^{-1}\right)$

$\begin{array}{lllll}3,170 & 0 & & & \\ 0 & 1,269 & \mathrm{~B}_{1} & & \\ & & & 4,523 & \mathbf{B}_{2}\end{array}$

$\begin{array}{rrr}6,598 & -2,023 & -2,888 \\ -2,023 & 5,001 & 1,822 \\ -2,888 & 1,822 & 4,706\end{array}$

Tableau VIII

Coefficients $l_{i \leqslant \sigma}^{y}$

\begin{tabular}{|c|c|c|c|c|c|c|c|c|c|c|c|c|c|}
\hline$i$ & $\alpha^{s \sigma}$ & $Q_{1}$ & $Q_{2}$ & $Q_{3}$ & $Q_{4}$ & $Q_{5}$ & $Q_{6}$ & $Q_{71}$ & $Q_{72}$ & $Q_{81}$ & $Q_{82}$ & $Q_{91}$ & $Q_{92}$ \\
\hline $\mathrm{Cl}$ & $\begin{array}{l}x \\
y \\
z\end{array}$ & \begin{tabular}{l|}
0 \\
0 \\
0,8136
\end{tabular} & $\begin{array}{l}0 \\
0 \\
0\end{array}$ & $\begin{array}{l}0 \\
0 \\
-0,2625\end{array}$ & $\begin{array}{l}0 \\
0 \\
0\end{array}$ & $\begin{array}{l}0 \\
0 \\
0\end{array}$ & $\begin{array}{l}0 \\
0 \\
0\end{array}$ & {$\left[\begin{array}{ll}-0,589 & 0 \\
-0,589 & 0 \\
0 & \end{array}\right.$} & $\mid \begin{array}{c}-0,589 \\
0,589 \\
0\end{array}$ & $\begin{array}{l}0,0474 \\
0,0474 \\
0\end{array}$ & $\mid \begin{array}{l}0,0474 \\
-0,0474 \\
0\end{array}$ & $\begin{array}{l}0,0728 \\
0,0728 \\
0\end{array}$ & $\begin{array}{r}0,0728 \\
-0,0728 \\
0\end{array}$ \\
\hline$F_{1}$ & $\begin{array}{l}x \\
y \\
z\end{array}$ & $\begin{array}{l}0,0237 \\
0 \\
-0,1529\end{array}$ & $\begin{array}{r}0,4940 \\
0 \\
-0,0345\end{array}$ & $\begin{array}{l}0,0735 \\
0 \\
0,2815\end{array}$ & $\begin{array}{l}0,4988 \\
0 \\
-0,0349\end{array}$ & $\begin{array}{l}0,0349 \\
0 \\
0,4988\end{array}$ & $\mid \begin{array}{l}0 \\
-0,500 \\
0\end{array}$ & $\begin{array}{r}0,1894 \\
0,1251 \\
-0,1027\end{array}$ & $\begin{array}{r}0,1894 \\
-0,1251 \\
-0,1027\end{array}$ & 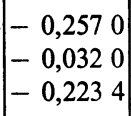 & 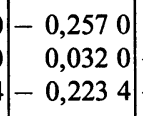 & $\begin{array}{r}0,2697 \\
-0,3975 \\
-0,0654\end{array}$ & $\begin{array}{r}0,2697 \\
0,3975 \\
-0,0654\end{array}$ \\
\hline$F_{2}$ & $\begin{array}{l}x \\
y \\
z\end{array}$ & $\begin{array}{l}0 \\
0,0237 \\
-0,1529\end{array} \mid$ & $\left|\begin{array}{r}0 \\
0,4940 \\
-0,0345\end{array}\right|$ & $\begin{array}{l}0 \\
0,0735 \\
0,2815\end{array}$ & $\begin{array}{l}0 \\
-0,4988 \\
0,0349\end{array}$ & $\left|\begin{array}{l}0 \\
-0,0349 \\
-0,4988\end{array}\right|$ & $\begin{array}{l}-0,500 \\
0 \\
0\end{array}$ & $\begin{array}{r}0,1251 \\
0,1894 \\
-0,1027\end{array}$ & $\begin{array}{r}0,1251 \\
-0,1894 \\
0,1027\end{array}$ & $\begin{array}{l}-0,032 \\
-0,257 \\
-0,223\end{array}$ & $\mid \begin{array}{rr}-0,032 & 0 \\
0,257 & 0 \\
0,223 & 4\end{array}$ & $\begin{array}{r}-0,3975 \\
0,2687 \\
-0,0654\end{array}$ & $\begin{array}{r}-0,3975 \\
-0,2697 \\
0,0654\end{array}$ \\
\hline $\mathrm{F}_{3}$ & $\begin{array}{l}x \\
y \\
z\end{array}$ & $\left|\begin{array}{l}-0,0237 \\
0 \\
-0,1529\end{array}\right|$ & $\begin{array}{l}-0,4940 \\
0 \\
-0,0345\end{array}$ & $\begin{array}{l}-0,0735 \\
0 \\
0,2815\end{array}$ & {$\left[\begin{array}{l}-0,4988 \\
0 \\
-0,0349\end{array}\right.$} & $\mid \begin{array}{l}-0,0349 \\
0 \\
0,4988\end{array}$ & \begin{tabular}{ll|l}
0 \\
0,500 & 0 \\
0
\end{tabular} & \begin{tabular}{ll|}
0,189 & 4 \\
0,125 & 1 \\
0,102 & 7
\end{tabular} & $\begin{array}{r}0,1894 \\
-0,1251 \\
0,1027\end{array}$ & $\begin{array}{r}-0,2570 \\
-0,0320 \\
0,2234\end{array}$ & $\mid \begin{array}{r}-0,2570 \\
0,0320 \\
0,2234\end{array}$ & $\begin{array}{r}0,2697 \\
-0,3975 \\
0.0654\end{array}$ & $\begin{array}{c}0,2697 \\
0,3975 \\
0,0654\end{array}$ \\
\hline $\mathrm{F}_{4}$ & $\begin{array}{l}x \\
y \\
z\end{array}$ & $\begin{array}{c}0 \\
-0,0237 \\
-0,1529\end{array}$ & $\begin{array}{l}0 \\
-0,4940 \\
-0,0345\end{array}$ & $\begin{array}{l}0 \\
-0,0735 \\
0,2815\end{array}$ & $\begin{array}{l}0 \\
0,4988 \\
0,0349\end{array}$ & $\begin{array}{l}0 \\
0,0349 \\
-0,4988\end{array}$ & $\begin{array}{l}0,500 \\
0 \\
0\end{array}$ & $\begin{array}{ll}0,125 & 1 \\
0,189 & 4 \\
0,102 & 7\end{array}$ & $\begin{array}{r}0,1251 \\
-0,1894 \\
-0,1027\end{array}$ & $\begin{array}{r}-0,0320 \\
-0,2570 \\
0,2234\end{array}$ & $\mid \begin{array}{r}-0,0320 \\
0,2570 \\
-0,2234\end{array}$ & $\begin{array}{r}-0,3975 \\
0,2697 \\
0,0654\end{array}$ & $\begin{array}{l}-0,3975 \\
-0,2697 \\
-0,0654\end{array}$ \\
\hline$F_{5}$ & $\begin{array}{l}x \\
y \\
z\end{array}$ & $\left|\begin{array}{l}0 \\
0 \\
-- \\
0.4921\end{array}\right|$ & $\begin{array}{l}0, \\
0 \\
0.1382\end{array}$ & $\begin{array}{l}0 \\
0 \\
0,7698\end{array}$ & $\begin{array}{l}0 \\
0 \\
0\end{array}$ & $\begin{array}{l}0 \\
0 \\
0\end{array}$ & $\begin{array}{l}0 \\
0 \\
0\end{array}$ & $\begin{array}{ll}0,170 & 1 \\
0,170 & 1 \\
0 & \end{array} \mid$ & $\left|\begin{array}{cc}0,170 & 1 \\
-0,170 & 1 \\
0 & \end{array}\right|$ & $\begin{array}{l}0,5136 \\
0,5136 \\
0\end{array}$ & $\left|\begin{array}{l}0,5136 \\
-0,5136 \\
0\end{array}\right|$ & $\begin{array}{l}0,1568 \\
0,1568 \\
0\end{array}$ & $\begin{array}{c}0,1568 \\
-0,1568 \\
0\end{array}$ \\
\hline
\end{tabular}

TABLEAU IX

Coefficients $a_{s \sigma}^{\alpha \beta}$

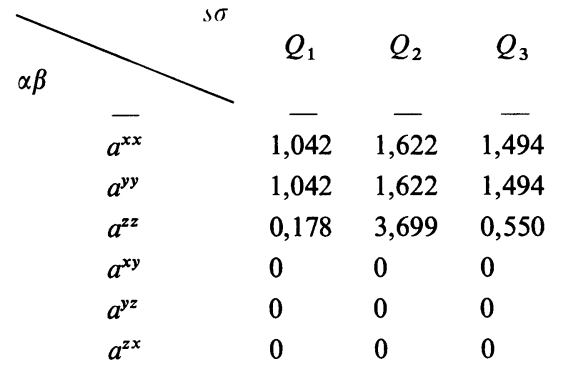

$\times 4,074 \times 10^{-34}\left(\mathrm{~kg} \times \mathrm{m}^{2} \times \mathrm{s}^{-1}\right)^{1 / 2}$

$\begin{array}{cclllllll}Q_{4} & Q_{5} & Q_{6} & Q_{71} & Q_{81} & Q_{91} & Q_{72} & Q_{82} & Q_{92} \\ - & - & - & - & - & - & - & - & - \\ -1,867 & -0,131 & 0 & 0 & 0 & 0 & 0 & 0 & 0 \\ 1,867 & 0,131 & 0 & 0 & 0 & 0 & 0 & 0 & 0 \\ 0 & 0 & 0 & 0 & 0 & 0 & 0 & 0 & 0 \\ 0 & 0 & 1,872 & 0 & 0 & 0 & 0 & 0 & 0 \\ 0 & 0 & 0 & 0,384 & 0,836 & 0,245 & -0,384 & -0,836 & -0,245 \\ 0 & 0 & 0 & 0,384 & 0,836 & 0,245 & 0,384 & 0,836 & 0,245\end{array}$


et $\theta_{0}$ ) permet de déterminer la position de l'atome de chlore sur l'axe de la molécule. Utilisant alors $\theta$ comme paramètre [10] le formalisme de Nielsen permet de calculer la valeur de $\mathbf{R}_{6}$; la valeur de $\theta$ retenue pour $\mathrm{ClF}_{5}$ est celle pour laquelle $R_{6 \text { calc }}=R_{6 \mathrm{mes}}$ aux incertitudes expérimentales près. Ceci permet de proposer

$$
\begin{aligned}
& \theta=86^{\circ} \pm 0,5^{\circ} \\
& R_{a x}=1,58 \pm 0,05 \AA \\
& R_{e q}=1,67 \pm 0,05 \AA \\
& C=\frac{h}{8 \Pi^{2} I_{z}}=2400 \pm 20 \mathrm{MHz} .
\end{aligned}
$$

Partant de ce résultat on procède de proche en proche (étude de $D_{J}$ puis de $D_{J K}$ ) pour déterminer éventuellement les éléments de la matrice $M$; on

\begin{tabular}{|c|c|c|}
\hline $\begin{array}{l}F_{i j} \times 10^{5} \\
(\text { dyne } / \mathrm{cm})\end{array}$ & $\begin{array}{c}\text { Présent } \\
\text { travail }\end{array}$ & $\frac{\partial F}{\partial \theta}$ \\
\hline- & - & - \\
\hline$F_{11}$ & 3,09 & 0,05 \\
\hline$F_{12}$ & $-0,02$ & 0,01 \\
\hline$F_{13}$ & 0,52 & 0,02 \\
\hline$F_{22}$ & 3,25 & 0 \\
\hline$F_{23}$ & 0,07 & 0,005 \\
\hline$F_{33}$ & 3,30 & 0,1 \\
\hline$F_{44}$ & 2,61 & 0 \\
\hline$F_{45}$ & 0 & 0 \\
\hline$F_{55}$ & 3,35 & 0,2 \\
\hline$F_{66}$ & 1,10 & 0,02 \\
\hline$F_{77}$ & 1,73 & 0,02 \\
\hline$F_{78}$ & 0,07 & 0,03 \\
\hline$F_{79}$ & $-0,26$ & 0,01 \\
\hline$F_{88}$ & 1,86 & 0,04 \\
\hline$F_{89}$ & 0,12 & 0,01 \\
\hline$F_{99}$ & 1,43 & 0,01 \\
\hline
\end{tabular}

\section{TABLEAU X}

Constantes de force de $\mathrm{ClF}_{5}$ (coordonnées symétriques internes) constate ici que si la précision recherchée dans les calculs théoriques est limitée à $5 \%$, il est inutile de considérer l'effet de cette matrice, ce qui justifie a posteriori le fait de prendre $L \equiv L_{0}(M=E)$ : le système de coordonnées symétriques choisi est donc très voisin du système des coordonnées normales. On peut alors donner les éléments de la matrice $G$ (Tableau VII), les coefficients $l_{i s \sigma}^{\alpha}$ (Tableau VIII) les coefficients $a_{s \sigma}^{\alpha \beta}$ (Tableau IX) ainsi que les éléments de la matrice $F$ (Tableau $\mathrm{X}$ ). Finalement dans le tableau XI nous comparons notre détermination des constantes de force pour $\mathrm{ClF}_{5}$ à celles publiées par ailleurs $[4,11,12]$.

\begin{tabular}{|c|c|c|c|c|}
\hline $\begin{array}{c}f \times 10^{5} \\
(\text { dyne } / \mathrm{cm})\end{array}$ & $\begin{array}{c}\text { Présent } \\
\text { travail }\end{array}$ & $\frac{\partial f}{\partial \theta}$ & Réf. [12] & Réf. [4] \\
\hline - & - & - & - & - \\
\hline$f_{\mathrm{R}}$ & 3,09 & 0,05 & 3,16 & 3,01 \\
\hline$f_{\mathrm{r}}$ & 2,33 & 0,01 & 2,46 & 2,57 \\
\hline$f_{\mathrm{ru}}$ & 0,16 & 0 & 0,17 & 0,17 \\
\hline$f_{\mathrm{rr}}^{\prime}$ & 0,60 & 0,01 & 0,46 & 0,33 \\
\hline$f_{\beta}$ & 2,60 & 0,1 & 3,09 & 3,11 \\
\hline$f_{\beta \beta}$ & $-0,01$ & 0,03 & 0,13 & \\
\hline$f_{\beta \beta}^{\prime}$ & 0,73 & 0,06 & 0,54 & 1,29 \\
\hline$f_{\alpha}^{p p}$ & 1,00 & 0,04 & 1,17 & 1,06 \\
\hline$f_{\alpha \alpha}$ & $-0,26$ & 0,02 & & \\
\hline$f_{\alpha \alpha}^{\prime}$ & $-0,43$ & 0,03 & $-0,02$ & \\
\hline$f_{\mathrm{Rr}}$ & 0,01 & 0,01 & & 0,25 \\
\hline$f_{\mathrm{R} \alpha}\left({ }^{*}\right)$ & 0,03 & & & \\
\hline$f_{\mathrm{R} \beta}$ & 0,26 & 0,01 & 0,29 & \\
\hline$f_{\mathrm{r} \beta}$ & 0,05 & 0,02 & & \\
\hline$f_{\mathrm{r} \beta}^{\prime}$ & 0,02 & 0,02 & & \\
\hline$f_{\mathbf{r} \beta}^{\prime \prime}$ & $-0,02$ & 0,02 & & \\
\hline$f_{\mathrm{r}}\left({ }^{*}\right)$ & $-0,09$ & & $-0,01$ & \\
\hline$f_{\mathrm{r} \alpha}^{\prime \prime}(*)$ & 0,09 & & 0,01 & \\
\hline$f_{\alpha \beta}\left({ }^{*}\right)$ & 0,15 & & & \\
\hline$f_{\alpha \beta}^{\prime}\left({ }^{*}\right)$ & 0,07 & & & \\
\hline
\end{tabular}

\section{TABLEAU XI}

Constantes de forces de $\mathrm{ClF}_{5}$ (coordonnées internes)

$\left(^{*}\right)$ Les valeurs obtenues par le calcul ne sont pas significatives.

Bibliographie

[1] Jurek, R., Suzeau, P., Chanussot, J., Champion, J. P., J. Physique 35 (1974) 533.

[2] Bradley, R. H., Brier, P. N., Whittle, M. J., J. Mol. Spectros. 44 (1972) 536

[3] Suzeau, P., Jurek, R., Chanussot, J., C. R. Hebd. Séan. Acad. Sci. 276-777 (1973)

[4] Begun, G. M., Fletcher, U. H., Smith, D. F., J. Chem. Phys. 42 (1965) 2236.

[5] Nielsen, H. H., Rev. Mod. Phys. 23 (1951) 90.
[6] Wilson, E. B., Decius, J. C., Cross, P. C., Molecular vibrations (Mc Graw Hill) 1955.

[7] Henry, L., Amat, G., Cah. Phys. 118 (1960) 230.

[8] KirchHoff, W. H., J. Mol. Spectros. 41 (1972) 333.

[9] Costain, C. C., J. Chem. Phys. 29 (1958) 864.

[10] Legon, A. C., J. Chem. Soc. Faraday Trans 269 (1973) 29.

[11] Ramaswamy, K., Muthusubramanian, P., J. Mol. Struct. 7 (1971) 45.

[12] Curtis, E. C., Spectrochim. Acta 27A (1971) 1989. 